\title{
Conducta alimentaria y perfil glucémico en dos líneas de ratas con diabetes genética: eSS y eSMT
}

\author{
Silvana Marisa Montenegro ${ }^{1,2}$, María Cristina Tarrés ${ }^{1,2}$, Juan Carlos Picena ${ }^{1}$, \\ Stella Maris Martínez ${ }^{1,2}$ \\ ${ }^{1}$ Facultad de Ciencias Médicas, Universidad Nacional de Rosario, Rosario, Argentina. \\ ${ }^{2}$ Consejo de Investigaciones, Universidad Nacional de Rosario, Rosario, Argentina.
}

Introducción. La alimentación puede agravar la diabetes de humanos y modelos animales. Entre ellos, la rata eSMT presenta un curso más exacerbado y mayor biomasa que la línea parental eSS.

Objetivo. Estudiar la conducta alimentaria de ratas macho eSMT y eSS ante oferta ilimitada y bajo restricción alternada, analizando su incidencia sobre el peso y la glucemia.

Materiales y métodos. Se registró el consumo ad libitum en etapas de crecimiento y mantenimiento. Un grupo de eSMT recibió durante 5 semanas a partir del destete, una dieta restringida intermitente seguida de oferta ilimitada, y otro fue restringido hasta el año de edad, midiéndose el peso y el perfil glucémico en distintas edades.

Resultados. Durante el crecimiento, eSMT aumentó de peso más que eSS con ingestas similares, y en la etapa de mantenimiento sustentó mayor biomasa con menor consumo. La restricción intermitente posterior al destete indujo un patrón de crecimiento más lento y menor peso adulto. A los 5 meses presentaron valores de $\mathrm{G} 0$ normales $(85 \pm 12 \mathrm{mg} / \mathrm{dl})$ y los controles mostraron cifras alteradas $(119 \pm 14 \mathrm{mg} / \mathrm{dl})$. En los animales restringidos la posterior oferta ilimitada produjo glucemias de ayuno compatibles con diabetes $(163 \pm 25 \mathrm{mg} / \mathrm{dl})$ y un incremento ponderado $75 \%$ superior a los alimentados ad libitum.

Conclusión. La alternancia prolongada hambre-saciedad indujo en eSMT desaceleración de su metabolopatía mientras que la realimentación se asoció con incrementos superiores de peso y glucemia, atribuibles a un aumento de la eficiencia de conversión inducida por el ayuno. Estos resultados enfatizan la importancia de mejorar el conocimiento sobre las relaciones entre crecimiento, alimentación y diabetes.

Palabras clave: diabetes mellitus, modelos animales, conducta alimentaria, peso corporal, glucosa de la sangre, ratas.

\section{Feeding behavior and glycemic profile in two lines of rats with genetic diabetes}

Introduction. Food intake can aggravate diabetes in humans and in animal models. In rat model systems, the eSMT rat strain shows increased diabetes expression and higher biomass than its eSS parental line.

Obvjective. The feeding behavior of eSMT and eSS males was compared under conditions of ad libitum versus restrictive food intake. The effects on rat weight and glycemia were examined. Materials and methods. Unrestricted food intake during growth and maintenance stages was recorded. One group of eSMT was submitted to intermittent dietary restriction for 5 weeks after weaning, followed by ad libitum food availability. A second group was restricted until one year of age. Weights and glycemic profiles were measured at periodic intervals.

Results. During the growth stage, eSMT showed weight increases higher than the eSS with similar food intake. In the maintenance stage, eSMT reached higher biomass with less intake. Post-weaning alternate restriction induced a slower growth pattern and lower adult weight. After five months of fasting, glycemia was normal $(85 \pm 12 \mathrm{mg} / \mathrm{dl})$ and controls showed altered values $(119 \pm 14 \mathrm{mg} / \mathrm{dl})$. Ad libitum nutrition after restriction resulted in glycemia levels compatible with diabetes $(163 \pm 25 \mathrm{mg} / \mathrm{dl})$ and a weight gain $75 \%$ higher than in ad libitum-fed animals.

Conclusions. Prolonged hunger-satiety alternation induced slowing of the eSMT metabolopathy, whereas refeeding was associated with higher increases of weight and glycemia.This was 
imputed to an augmented conversion efficiency caused by fasting. These results emphasize the importance of understanding the relations between growth, feeding and diabetes.

Key words: Diabetes mellitus, animal models, animal feeding behavior, body weight, blood glucose, rats.

La diabetes mellitus representa un grupo de enfermedades etiológica y clínicamente heterogéneo, caracterizado por hiperglucemia crónica y otras anormalidades metabólicas, debidas a una deficiencia en la acción de la insulina (1). Para la investigación de los diversos tipos de diabetes se han utilizado ampliamente distintas especies animales y los resultados obtenidos han contribuido de modo significativo al conocimiento actual de variados aspectos de la enfermedad $(2,3)$.

El Comité de Expertos en Diabetes de la OMS ha señalado la necesidad del empleo de modelos animales y de su desarrollo, dado que permiten investigar la fisiopatología del síndrome diabético imitando la complejidad del ser humano $(4,5)$. Más aún, últimamente se los recomienda para la realización de estudios terapéuticos que permitan su posterior valoración clínica en el control dietético de pacientes (6).

Los modelos murinos eSS, B y eSMT, pertenecientes a la Facultad de Ciencias Médicas de la Universidad Nacional de Rosario, Argentina, son cepas de ratas endocriadas derivadas de un núcleo fundacional común inscrito con el nombre IIM en 1964 y registrado nuevamente en 1993 (7).

Los animales B muestran sobrepeso respecto de la línea original IIMb, obesidad moderada que se acompaña de hipertrigliceridemia y, en forma tardía, intolerancia a la glucosa $(8,9)$. La rata eSS es un modelo no obeso de diabetes espontánea tipo 2 con manifestaciones bioquímicas e histopatológicas progresivas con la edad, y de mayor gravedad en machos $(10,11)$. Estos animales no exhiben un acortamiento importante de su vida, ya que sobreviven hasta más de los 18 meses sin administración exógena de insulina,

$\overline{\text { Correspondencia: }}$

Silvana M Montenegro.

Bv Oroño 1505.1…ㅇ..

Tel: 054-341-4474082

Email: smontene@fmedic.unr.edu.ar

Recibido: 11/04/05; aceptado:27/07/05 hecho que ha contribuido para que esta línea haya sido reconocida como modelo biológico para el estudio de la diabetes tipo 2 y sus complicaciones crónicas (12-15).

La rata eSMT es una línea recombinante o sintética obtenida por el cruce de eSS con B y posterior endocría. Los animales muestran comienzo anticipado y exacerbación de dos características centrales del síndrome diabético: hiperglucemia de ayuno e intolerancia a la glucosa y, por sus características metabólicas, constituye un modelo de la diabetes tipo 2 con sobrepeso (16).

Se sabe que los síndromes diabéticos tipo 2, tanto en el hombre como en animales, están fuertemente influidos por las variaciones del peso, habiéndose determinado que una mayor biomasa en la adultez es capaz de agravar, o aún de inducir, alteraciones en la homeostasis glúcida $(17,18)$.

En la línea eSS, el peso, factor determinante de la intensidad de la metabolopatía, se modifica con el ambiente nutricional, de tal modo que las restricciones calóricas continuas o intermitentes disminuyen la expresión del síndrome diabético (19-21). Además, las dietas con menor contenido de grasa y proteína disminuyen las alteraciones bioquímicas y retrasan la aparición de la organopatía mientras que, por el contrario, el consumo irrestricto de sacarosa las empeora $(19,22)$.

En función de lo expuesto, resultaba de interés estudiar en la línea eSMT la conducta frente a una oferta ad libitum o alternada de alimento en comparación con la línea parental eSS, su efecto sobre el crecimiento, peso adulto y longitud caudal, así como analizar su vinculación con el perfil glucémico.

\section{Materiales y métodos}

Se emplearon machos eSMT y eSS provenientes del bioterio de la Cátedra de Biología de la Facultad de Ciencias Médicas de la UNR, donde las condiciones de cría incluyen regulación de la temperatura $\left(24^{\circ} \mathrm{C}\right)$ y de los ciclos luz-oscuridad, así como el recambio de aire, provisión de agua 
ad libitum y alimento balanceado comercial especial para roedores de laboratorio.

En todos los experimentos, las muestras de sangre se obtuvieron por punción de las venas de la cola, método de recolección recomendado para la especie porque causa poco dolor y no daña al animal (23). Las dosificaciones se efectuaron a la misma hora por la mañana para evitar los efectos de posibles ritmos circadianos. La glucosa se suministró por sonda gástrica en solución al $10 \%$ (200 mg/100 g de peso corporal) y la glucemia se determinó con un método enzimático colorimétrico.

Para la caracterización clínica de la diabetes se empleó el valor de la glucemia, medida en condiciones de ayuno y en respuesta a la sobrecarga con glucosa. La hiperglucemia en dichas condiciones es una forma aceptada para definir diabetes y sus valores usualmente se emplean en la detección primaria y el diagnóstico de la enfermedad (2).

\section{Experimento 1: consumo ad libitum}

Se analizó el consumo ad libitum de 10 machos eSMT y 9 eSS en dos períodos: el primero coincidiendo con la etapa de crecimiento y el segundo con la etapa de mantenimiento.

\section{Primer período}

A los 40 días de edad, se los colocó en jaulas individuales de metabolismo durante 2 semanas y se midió diariamente el peso y la ingesta. Al iniciar y finalizar el período se registró el largo de la cola, indicador del largo del esqueleto (24).

Se calcularon el peso medio (PM), el incremento de peso $(\Delta \mathrm{P})$, el incremento de largo de cola $(\Delta \mathrm{L})$, el consumo medio diario (CMD), el consumo medio diario relativo $=\mathrm{CMD} /$ peso $\times 100\left(\mathrm{CMD}_{\mathrm{R}}\right)$ y el consumo calórico (CCal).

Se compararon el peso inicial, el peso final, el $\Delta \mathrm{P}$, el $\mathrm{PM}$, los largos de cola inicial y final, el $\Delta \mathrm{L}$, el CMD, el $C M D_{R}$ y el CCal entre las líneas.

\section{Segundo período}

A los 6 meses de edad, los animales fueron evaluados de idéntica forma que en el primer período, calculándose el PM, el largo de cola medio ( $L M)$, el CMD, el $C M D_{R}$ y el CCal, variables que fueron comparadas entre las líneas.

\section{Experimento 2: restricción alternada de alimento}

Se denominó de esta forma un régimen consistente en alimentación ad libitum dos días $y$ ayuno en el tercero (21). Los grupos experimentales fueron los que se describen en las siguientes secciones.

Cinco semanas de restricción a partir del destete

De un lote de 18 machos eSMT, 11 se restringieron desde los 21 hasta los 56 días de edad (R), en tanto que los 7 restantes actuaron como controles recibiendo alimentación ad libitum (AL). Al finalizar este lapso, todos continuaron con el mismo alimento sin restricciones, divididos en los grupos de realimentado (R-AL) y ad libitum $(A L)$. Se midió el peso semanalmente desde los 21 hasta los 56 días y a los 70 días.

Para estudiar el crecimiento posterior al destete, los datos se ajustaron mediante el modelo de von Bertalanffy, cuya ecuación es :

$\mathrm{P}(\mathrm{t})=\mathrm{A}\left(1-\mathrm{be} \mathrm{e}^{-\mathrm{kt}}\right)^{3}$ donde:

$\mathrm{P}(\mathrm{t})$ : peso ( $\mathrm{g}$ ) en el tiempo $\mathrm{t}$ (días)

A: peso asintótico, valor de $\mathrm{P}(\mathrm{t})$ cuando $\mathrm{t} \rightarrow \infty$

b: parámetro de escala

k: tasa de maduración

t: edad (días)

Según este modelo, el peso se aproxima a una asíntota cuyo valor es el del parámetro "A"; la tasa de maduración o " $k$ " es una constante proporcional al crecimiento total y define la tasa de crecimiento del animal mientras que "b" carece de interpretación biológica $(25,26)$.

Se calcularon las tasas de madurez y el peso asintótico en el período entre los 21 y 56 días y el incremento de peso entre los 56 y 70 días.

A los 6 meses, se determinó el peso y las glucemias de ayuno (G0) y a los 30 (G30), 60 (G60) y 120 minutos (G120) después de una sobrecarga oral con glucosa, así como la glucosuria registrada en la orina emitida por 
micción espontánea durante la ejecución de la curva de tolerancia glúcida.

\section{Restricción prolongada a partir del destete}

Se aplicó el mismo esquema de restricción en machos eSMT y se lo prolongó hasta los 11 meses de edad (grupo $R, n=10$ ), empleando controles de la misma línea (grupo $A L, n=10$ ) con alimentación ad libitum.

Los animales fueron colocados en jaulas de metabolismo a los 4 y a los 9 meses de edad. Luego de un período de acostumbramiento de 5 días, durante 10 días se midieron la ingesta y el peso, calculando el consumo total (CT), el peso promedio, el consumo total relativo $=\mathrm{CT} /$ peso $\left(\mathrm{CT}_{\mathrm{R}}\right)$, y el CMD.

A los 5 y 11 meses se registraron el peso $(P), G 0$ y G120.

\section{Análisis estadístico}

Los datos fueron procesados mediante el software SPSS for Windows, Release 8.0, SPSS Inc, 1995, utilizándose pruebas paramétricas para muestras independientes o relacionadas que se indican en cada caso en la presentación de resultados.

En todos los casos se consideró significativo un valor de $p<0,05$.

\section{Evaluación bioética}

El protocolo de investigación fue examinado por la Comisión de Bioética de la Facultad de Ciencias Médicas de la UNR, la cual lo consideró aceptable desde el punto de vista ético.

\section{Resultados}

\section{Experimento 1: consumo ad libitum}

Las comparaciones entre genotipos efectuadas durante el primer período de estudio se presentan en el cuadro 1.

Si bien no existieron diferencias estadísticamente significativas entre líneas en el peso al comienzo y final del período, se comprobó que en esta etapa temprana de crecimiento, eSMT tuvo un aumento ponderal mayor que eSS, sin que se evidenciaran en esta edad diferencias en el tamaño del esqueleto ni en el consumo de alimento.
Al examinar el segundo período, esta similitud no se mantiene. Aunque la longitud caudal de eSMT no mostró diferencias significativas con eSS (eSMT: $18,2 \pm 0,3 \mathrm{~cm}$ vs. eSS: $17,8 \pm 0,4 \mathrm{~cm}$, $p>0,05$ ), el peso medio fue sensiblemente mayor en la línea sintética (eSMT: $374 \pm 33 \mathrm{~g}$ vs. eSS: $298 \pm 18 \mathrm{~g}, p<0,01$ ), siendo interesante destacar que eSMT logró un peso superior con menor consumo de alimento (CMD = eSMT: $24,4 \pm 1,5 \mathrm{~g}$ vs. eSS: $28,7 \pm 1,3 \mathrm{~g}, p<0,001 ; \mathrm{CMD}_{\mathrm{R}}=\mathrm{eSMT}$ :

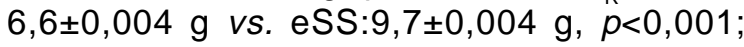
Ccal=eSMT: $74 \pm 5 \mathrm{kcal} / \mathrm{día}$ vs. eSS: $88 \pm 4 \mathrm{kcal} /$ día, $p<0,001)$.

Para estudiar la conducta de cada línea entre períodos, se efectuaron comparaciones apareadas. En eSMT, el peso medio aumentó significativamente entre los 40 días y los 6 meses $(t=13,07, p<0,001)$, en tanto que las variables relacionadas con el consumo disminuyeron de valor (CMD: $t=-3,33, p<0,05 ; C_{R}: t=-13,65$, $p<0,001$; CCal: $t=-3,33, p=0,05)$. En eSS el incremento significativo del peso medio $(t=11,53$, $p<0,001)$ fue acompañado de mayor $\operatorname{CMD}(t=3,49$, $p<0,05)$, superior ingesta calórica $(t=3,49, p<0,05)$ y menor $\mathrm{CMD}_{\mathrm{R}}(t=-4,62, p<0,01)$. La reducción en el $C M D_{R}$ se debe no sólo al aumento del denominador sino a la disminución en el consumo ya mencionada, lo que implica que a esta edad, eSMT puede mantener mayor biomasa con menor ingesta calórica.

En resumen, en el primer período, eSMT registró un aumento de peso mayor que eSS con un consumo de alimento similar, en tanto que en el segundo período, eSMT mantuvo una mayor biomasa con una menor ingestión calórica.

\section{Experimento 2: restricción alternada de alimento}

Cinco semanas de restricción a partir del destete

Al efectuar la restricción alimentaria alternada durante 5 semanas a partir del destete, se alcanzaron los resultados que se exponen en el cuadro 2.

Si bien en el momento de comenzar la restricción los pesos de ambos grupos eran similares, al finalizarla los valores eran visiblemente mayores en el grupo control, verificándose un aumento en el grupo restringido de $52 \%$ respecto del 
Cuadro 1. Peso, largo de cola y consumo ad libitum medidos en un período de dos semanas a partir de los 40 días de edad en machos eSMT y eSS.

\begin{tabular}{|c|c|c|c|c|c|c|c|c|c|c|}
\hline Línea & $\begin{array}{c}\text { Peso } \\
\text { inicial }(g)\end{array}$ & $\begin{array}{c}\text { Peso } \\
\text { final g) }\end{array}$ & $\Delta P(g)$ & $\begin{array}{c}\text { Peso } \\
\text { medio (g) }\end{array}$ & $\begin{array}{l}\text { Largo de cola } \\
\text { inicial }(\mathrm{cm})\end{array}$ & $\begin{array}{l}\text { Largo de cola } \\
\quad \text { final }(\mathbf{c m})\end{array}$ & $\begin{array}{c}\Delta \mathrm{L} \\
(\mathrm{cm})\end{array}$ & $\begin{array}{l}\text { CMD } \\
(\mathrm{g})\end{array}$ & $\begin{array}{l}\mathrm{CMD}_{\mathrm{R}} \\
(\mathrm{g})\end{array}$ & $\begin{array}{c}\text { CCal } \\
\text { (kcal/día) }\end{array}$ \\
\hline eSMT & $139 \pm 28$ & $282 \pm 33$ & $143 \pm 08$ & $216 \pm 31$ & $13,1 \pm 1,1$ & $16,1 \pm 1,2$ & $2,9 \pm 1$ & $26,5 \pm 3$ & $12,2 \pm 0,8$ & $81 \pm 8$ \\
\hline eSS & $154 \pm 10$ & $264 \pm 08$ & $110 \pm 05$ & $214 \pm 09$ & $13,6 \pm 0,7$ & $16,7 \pm 0,8$ & $3,0 \pm 1$ & $25,3 \pm 2$ & $12,0 \pm 1,2$ & $77 \pm 6$ \\
\hline$p$ & $>0,05$ & $>0,05$ & $<0,001$ & $>0,05$ & $>0,05$ & $>0,05$ & $>0,05$ & $>0,05$ & $>0,05$ & $>0,05$ \\
\hline
\end{tabular}

Valores expresados como media \pm desvío estándar. Prueba estadística: $t$ de student para muestras independientes.

Cuadro 2. Pesos al comienzo y al finalizar una restricción alternada de alimento en machos eSMT y parámetros de la curva von Bertalanffy.

\begin{tabular}{lccccc}
\hline Grupo & \multicolumn{2}{c}{ Peso $\mathbf{( g )}$} & & \multicolumn{2}{c}{ Parámetros von Bertalanffy } \\
\cline { 2 - 3 } \cline { 5 - 6 } & $\mathbf{2 1}$ días & 56 días & & Tasa de madurez & Peso asintótico \\
\hline Restringidos $(\mathrm{R})$ & $33,1 \pm 6,8$ & $124,7 \pm 22,4$ & & $0,016 \pm 0,006$ & $443,2 \pm 128,0$ \\
Control (AL) & $31,6 \pm 5,5$ & $212,4 \pm 22,7$ & & $0,024 \pm 0,006$ & $581,1 \pm 158,8$ \\
$p$ & $>0,05$ & $<0,001$ & & $<0,05$ & $<0,05$ \\
\hline
\end{tabular}

Valores expresados como media \pm desvío estándar. Prueba estadística: $t$ de student para muestras independientes.

alimentado ad libitum (R: $90,99 \pm 25,15 \mathrm{~g}$ vs. AL: $173,85 \pm 18,01 \mathrm{~g} ; p<0,001)$.

El patrón de crecimiento fue diferente, ya que la velocidad de crecimiento resultó menor en los animales bajo restricción alimenticia y el modelo aplicado predijo en ellos menor peso asintótico.

A partir de los 57 días de edad, cuando todos los animales fueron alimentados ad libitum, la evaluación efectuada a los 70 días mostró que el grupo realimentado, si bien mantuvo una diferencia de biomasa con el control (R-AL: $226,2 \pm 32,6$ g vs. AL: $270,6 \pm 19,7 ; p<0,05)$, logró un aumento superior (R-AL: $101,5 \pm 19,6$ vs. AL: $58,1 \pm 11,1 ; p<0,001)$.

Es interesante puntualizar que la recuperación del peso por parte de los animales eSMT previamente restringidos fue notoria, y si bien no pudieron igualar en tan corto plazo el de los controles, el incremento alcanzado fue $75 \%$ mayor en ese grupo.

A los 6 meses de edad, aún bajo alimentación ad libitum, los datos de la biomasa y la curva de tolerancia a la glucosa permiten observar las diferencias del grupo realimentado con respecto al control (cuadro 3).

Los machos que sufrieron la restricción alimentaria en una etapa temprana de su vida superaron ampliamente a los animales que contaron durante toda su vida con una alimentación irrestricta en cuanto a peso y al valor de la glucemia en los distintos momentos de la curva de tolerancia.

Siguiendo los criterios de la Asociación Americana de Diabetes, el grupo control se ajusta a la categoría de diagnóstico provisional de diabetes (G120 $\geq 200 \mathrm{mg} / \mathrm{dl}$ ), aunque su glucemia de ayuno es normal, mientras que el grupo restringido responde al mismo diagnóstico pero con valores de G0 correspondientes a la categoría de diabético ( $\geq 126 \mathrm{mg} / \mathrm{dl}$ ) y de $\mathrm{G} 120$ ostensiblemente superiores a los valores mínimos de tal categoría (2).

\section{Restricción prolongada a partir del destete}

En las evaluaciones en jaulas de metabolismo, el esquema alimentario de hambre-saciedad, aplicado en forma ininterrumpida desde el destete hasta los 11 meses de edad en otro grupo de animales eSMT, produjo los resultados que se resumen en el cuadro 4.

A los 4 meses de edad, los animales restringidos y los que se alimentaron ad libitum consumieron la misma cantidad de alimento si consideramos el período completo. Al relacionar este consumo con el peso del animal y al calcular el consumo medio diario, los valores de los restringidos fueron 
Cuadro 3. Peso, glucemias y glucosuria en machos eSMT realimentados y controles a los 6 meses de edad.

\begin{tabular}{lcccccc}
\hline Grupo & $\begin{array}{c}\text { Peso } \\
\text { (g) }\end{array}$ & $\begin{array}{c}\text { G0 } \\
\text { (mg/d) }\end{array}$ & $\begin{array}{c}\text { G30 } \\
(\mathbf{m g} / \mathbf{d l})\end{array}$ & $\begin{array}{c}\text { G60 } \\
(\mathbf{m g} / \mathbf{d l})\end{array}$ & $\begin{array}{c}\text { G120 } \\
\text { (mg/dl) }\end{array}$ & $\begin{array}{c}\text { Glucosuria } \\
\text { (mg/dl) }\end{array}$ \\
\hline Realimentado (R-AL) & $466,0 \pm 31,4$ & $162,9 \pm 25,0$ & $470,4 \pm 92,8$ & $510,4 \pm 78,7$ & $335,0 \pm 117,3$ & $510,0 \pm 376,1$ \\
Control ( AL ) & $403,1 \pm 16,1$ & $92,7 \pm 17,9$ & $291,7 \pm 66,2$ & $388,7 \pm 126,6$ & $200,3 \pm 14,0$ & $430,7 \pm 309,5$ \\
$p$ & $<0,001$ & $<0,001$ & $<0,001$ & $<0,05$ & $<0,01$ & $>0,05$ \\
\hline
\end{tabular}

Valores expresados como media \pm desvío estándar. Prueba estadística: $t$ de student para muestras independientes.

Cuadro 4. Peso y consumo evaluado en un período de 10 días a los 4 y 9 meses de edad en animales eSMT restringidos en forma prolongada y controles.

\begin{tabular}{|c|c|c|c|c|c|}
\hline Edad & Grupo & $\begin{array}{l}\text { Peso } \\
\text { (g) }\end{array}$ & $\begin{array}{l}C T \\
(g)\end{array}$ & $\begin{array}{l}\mathrm{CT}_{\mathrm{R}} \\
(\mathrm{g})\end{array}$ & $\begin{array}{c}\text { CMD } \\
(\mathrm{g})\end{array}$ \\
\hline \multirow[t]{2}{*}{4 meses } & $\mathrm{R}$ & $\underset{* * \star}{307 \pm 36}$ & $\begin{array}{c}205 \pm 18 \\
n s\end{array}$ & $\underset{*}{0,67 \pm 0,1}$ & $\underset{* *}{30 \pm 3}$ \\
\hline & $A L$ & $424 \pm 30$ & $229 \pm 35$ & $0,54 \pm 0,1$ & $23 \pm 4$ \\
\hline \multirow[t]{2}{*}{9 meses } & $\mathrm{R}$ & $\begin{array}{c}404 \pm 27 \\
n s\end{array}$ & $\underset{* * \star}{174 \pm 40}$ & $\underset{* \star \star}{0,43 \pm 0,1}$ & $\begin{array}{c}25 \pm 6 \\
\mathrm{~ns}\end{array}$ \\
\hline & $A L$ & $373 \pm 72$ & $276 \pm 26$ & $0,75 \pm 0,1$ & $28 \pm 3$ \\
\hline
\end{tabular}

Valores expresados como media \pm desvío estándar.

Prueba estadística: $t$ de student para muestras independientes. ns: no significativo; ${ }^{\star} p<0,05 ;{ }^{* \star} p<0,01 ;{ }^{* \star *} p<0,001$.

superiores, lo que indicaría una ingesta compensadora en los días siguientes al ayuno. Por el contrario, a los 9 meses los animales sin restricción tuvieron mayor consumo total y relativo de alimento, a pesar de lo cual sufrieron pérdida de peso.

El consumo medio diario de los restringidos no difirió entre las dos edades $(t=1,75 ; p>0,05)$, pero sí lo hicieron el CMD relativo $(t=5,92 ; p<0,01)$ y el peso promedio $(t=11,76 ; p<0,001)$. El otro grupo mostró únicamente valores distintos de CMD relativo entre períodos $(t=7,15 ; p<0,01)$.

Las glucemias $(\mathrm{mg} / \mathrm{dl})$ de ayuno y a los $120 \mathrm{~min}$. de sobrecarga glúcida fueron menores en los animales restringidos tanto a los 5 meses $(\mathrm{G} 0=$ R: $85 \pm 12$ vs. AL: $119 \pm 14, p<0,001 ; \mathrm{G} 120=\mathrm{R}$ : $81 \pm 7$ vs. AL: $143 \pm 24, p<0,001)$ como a los 11 meses de edad (G0 = R: $134 \pm 14$ vs. AL: $252 \pm 68$, $p<0,01 ; \mathrm{G} 120=\mathrm{R}: 203 \pm 59$ vs. AL: $472 \pm 115$, $p<0,01)$.

Al confrontar estos datos con los criterios de la Asociación Americana de Diabetes ya mencionados, se comprueba que este grupo se ajusta a la categoría de diagnóstico provisorio de diabetes (G0 $\square 126 \mathrm{mg} / \mathrm{dl}$ y $\mathrm{G} 120 \square 200 \mathrm{mg} / \mathrm{dl}$ ) a los 11 meses, mientras que el grupo alimentado ad libitum presenta glucemia de ayuno y posterior a la sobrecarga alteradas $(110 \square \mathrm{G} 0<126 \mathrm{mg} / \mathrm{dl}$ y $140 \mathrm{mg} / \mathrm{dl} \square \mathrm{G} 120<200 \mathrm{mg} / \mathrm{dl}$ ) a los 5 meses (2). Si bien todos pertenecen a la categoría de diabéticos a los 11 meses de edad, es interesante destacar que la G0 aumentó en forma mucho más notable en los animales del grupo no restringido.

En suma, los resultados aquí presentados indican que la alternancia prolongada de hambre-saciedad produce en machos del genotipo eSMT una desaceleración de su metabolopatía.

\section{Discusión}

Se estudió la respuesta de los animales de la línea eSMT a una dieta ad libitum y bajo restricción calórica, dada la influencia que el consumo de alimento tiene en la diabetes humana (27).

Bajo las condiciones habituales de alimentación ad libitum, el análisis de las variaciones del peso y del consumo indicó una mayor eficiencia de conversión del alimento en eSMT que en eSS, tanto durante el período de crecimiento como en la etapa de estabilización adulta. Así, en la fase puberal, eSMT mostró un aumento de peso mayor que eSS con un consumo de alimento similar y 
más adelante mantuvo una mayor biomasa con menor ingesta calórica. Este hallazgo resulta de importancia si se tiene en cuenta que, a su vez, ha sido comprobada mayor eficiencia en machos eSS que en controles eumetabólicos (20).

A partir de la observación del brusco aumento de incidencia de diabetes tipo 2 en grupos humanos de cazadores recolectores y de agricultores durante el proceso de incorporación rápida de la alimentación, y del estilo de vida sedentario "occidentales", Neel sugirió que en estas poblaciones prevalecía un "genotipo eficiente" (28). Uno de los mecanismos propuestos para explicar tal hipótesis es que, debido a una menor densidad de receptores insulínicos en hígado y músculo, y a la consecuente resistencia a la insulina en dichas células, el genotipo "ahorrativo" o "frugal" promueve un almacenamiento más eficiente de reservas en forma de grasa en los momentos de abundancia de alimento (29). Disponer de depósitos de grasa es beneficioso durante la escasez, en un escenario alternante de abundancia y privación, condiciones que habrían prevalecido durante muchísimas generaciones; pero si el acceso al alimento abundante se hace continuo, el genotipo eficiente se torna desadaptativo frente al ambiente, ya que induce el desarrollo de resistencia a la insulina y redunda en hiperglucemia. El páncreas responde con sobreproducción de insulina, lo que conducirá al proceso escalonado de obesidad y diabetes conocido como "diabesidad" (30). La capacidad compensatoria del páncreas es variable tanto en humanos como en modelos animales, pero a medida que claudican las células beta, se instala una diabetes manifiesta y progresiva (31).

En roedores silvestres se ha comprobado la presencia de rasgos de eficiencia y, particularmente, Psammomys obesus que se torna obeso y diabético al ser trasladado de su hábitat desértico natural a las condiciones de cría y alimentación del laboratorio, se considera un excelente modelo animal del genotipo "ahorrativo" de la diabetes humana tipo 2 (32-34).

Caracterizado el comportamiento de la línea eSMT con alimentación ad libitum, se analizó su respuesta frente a la manipulación de la dieta. La restricción dietética de 5 semanas desde el destete indujo un patrón de crecimiento más lento, que de acuerdo con el modelo de von Bertalanffy conduciría a un peso asintótico menor, siendo el peso realmente alcanzado por eSMT al cabo de este período de restricción muy inferior al de los animales control.

Es notable la diferencia de la evolución del síndrome diabético de eSMT según se mantenga o se suspenda la restricción. En los animales en que la restricción se prolongó hasta cerca del año, los menores valores de glucemia, particularmente de ayuno, junto a la menor biomasa, indican un mejor desempeño del genotipo diabético en ambientes "pobres". Es interesante señalar que a pesar de tener menor consumo total y relativo de alimento, los machos eSMT sometidos a restricción desde los 6 meses no mostraron la caída ponderal propia de los animales con alimentación ad libitum, lo que debe considerarse un signo de deterioro orgánico. Esto coincide con lo demostrado en machos eSS, que bajo alimentación restringida permanente presentaron un curso más benigno de su síndrome metabólico y de la progresión de las lesiones morfológicas (21).

En el mismo sentido, la restricción calórica continua de $30 \%$ desde el mes de edad y durante 5 semanas previno en la rata OLEFT, obesa y diabética tipo 2, la aparición de diabetes, probablemente debido a una disminución de la resistencia a la insulina (35). En la rata ZDF, también obesa y diabética, los animales bajo restricción calórica se mantuvieron euglicémicos, aunque con niveles altos de insulinemia, y no se produjo pérdida de células beta del islote como en los controles (36).

Estos resultados beneficiosos de la restricción calórica aplicada en cepas de roedores diabéticos no coinciden con lo demostrado en ratas Sprague Dawley no diabéticas, en las que una restricción calórica continua de $30 \%$ durante la pubertad indujo menor producción de insulina probablemente por una alteración en la capacidad productora del páncreas debida a la desnutrición (29). Por el contrario, en la rata hembra Goto Kakizaki, modelo de diabetes tipo 2, la aplicación en animales de un mes de edad de una restricción de 35\% no afectó la producción de insulina (37). 
Estos hallazgos señalan la mejor capacidad de los genotipos diabéticos para resistir los efectos adversos de la carencia de alimentos.

La restricción intermitente prolongada fue beneficiosa para las ratas eSMT. Sin embargo, la realimentación de los individuos previamente restringidos desde el destete indujo una acelerada recuperación de su biomasa, así como efectos más deletéreos sobre la homeostasis glúcida que la alimentación ad libitum continua. Podría suponerse que la limitación alimenticia durante un período temprano de la vida pudo afectar el número de receptores insulínicos. En este sentido, se ha comprobado un mayor riesgo de desarrollar diabetes tipo 2 en humanos con bajo peso al nacer, así como en crías de ratas alimentadas con una dieta hipoproteica durante la preñez, vinculado con la menor expresión de proteínas receptoras de insulina en el tejido muscular (38).

El período de restricción podría haber inducido en eSMT la puesta en marcha de mecanismos de adaptación al ayuno con aumento de la eficiencia metabólica, lo que explicaría la mayor ganancia de peso respecto de los controles al cabo de dos semanas de realimentación. Este enfoque podría ser relevante en la prevención y tratamiento de la diabetes humana, ya que es conocida la dificultad que tienen los pacientes para mantener dietas restringidas durante períodos prolongados (39). En quienes habían sido sometidos a restricción, el incremento posterior del consumo calórico se acompañó de aumento de la glucemia aun en aquéllos que no recuperaron el peso perdido (40).

La diabetes tipo 2 y la obesidad no son detectables al nacer sino que se manifiestan posteriormente, por lo que se los denomina "fenotipos tardíos" (41). Resulta atractiva la posibilidad de prevenirlas actuando en la infancia, para lo que es necesario mejorar el conocimiento de sus relaciones con el crecimiento y la alimentación, procurando identificar los momentos críticos en que los factores ambientales adquieren particular relevancia $(42,43)$.

\section{Conflicto de intereses}

Los autores del manuscrito declaran que no existen conflicto de intereses con Laboratorios Wiener ni con la Fundación San Cristóbal.

\section{Financiación}

Se agradece a los Laboratorios Wiener, a la Fundación San Cristóbal y a la Universidad Nacional de Rosario el aporte a la financiación de este trabajo.

\section{Referencias}

1. Kuzuya T, Nakagawa S, Satoh J, Kanazawa Y, Iwamoto T, Kobayashi M et al. Report of the Committee on the classification and diagnostic criteria of diabetes mellitus. Diabetes Res Clin Pract 2002;55: 65-85.

2. American Diabetes Association. Diagnosis and classification of diabetes mellitus. Diabetes Care 2004; 27(Suppl.1):5-10.

3. Bertram CE, Hanson MA. Animal models and programming of the metabolic syndrome. Br Med Bull 2001;60:103-21.

4. Grupo de Estudio de la OMS. Investigación y evolución futura. En: Organización Mundial de la Salud, editora. Diabetes mellitus. Serie de Informes Técnicos 727. Madrid: Gráficas Reunidas; 1985. p.94-8.

5. van Zutphen LFM. Laboratory animal science: a view on the edge of the millenium. Revista de Ciència 1999; 23-24:9.

6. Ramos RR, Flores JL, Téllez LE, Rivas JF, Alarcón FJ. Estudio experimental de la acción antihiperglucemiante del nopal (Opuntia streptacantha Lemaire). Animales de Experimentación 2000;5:17-21.

7. Festing $\mathbf{M}$. Abbreviated list of inbred strains of rats. En: Festing M, editor. International index of laboratory animals. Carshalton, Surrey: Lion Litho Limited; 1993. p.51-67.

8. Calderari SA, González AC, Gayol MC. Spontaneous hypertriglyceridemic obesity and hyperglycemia in an inbred line of rats. Int J Obes 1987;11:571-9.

9. Calderari SA, Gayol MC, Ellif M, Labourdette VB, Troiano MF, Romano G. Desarrollo peripuberal de la obesidad en ratas B. Variaciones diarias de ingestión, peso, temperatura corporal profunda, trigliceridemia y glucemia. Acta Physiol Pharmacol Latinoam 1990;40: 391-402.

10. Martínez SM, Tarrés MC, Montenegro SM, Milo R, Picena JC, Figueroa $\mathbf{N}$ et al. Spontaneous diabetes in eSS rats. Acta Diabetol Lat 1988;25:303-13.

11. Tarrés MC, Martínez SM, Montenegro SM, Figueroa NS, D'Ottavio AE, Picena JC. Influence of gonadectomy in eSS diabetic rats. Rev Esp Fisiol 1997; 53:211-6.

12. Tarrés MC, Martínez SM, Montenegro SM, Picena JC, Llorens A, Naves A. The eSS rat. A model of noninsulin-dependent human diabetes. Am J Pathol 1992; 141:761-3. 
13. Martínez SM, Tarrés MC, Picena JC, Montenegro SM, Gagliardino JJ, Gómez Dumm CL et al. eSS rat, an animal model for the study of spontaneous noninsulin-dependent diabetes. En: Shafrir E, editor. Lessons from animal diabetes IV. London: SmithGordon and Company Limited Editors; 1993. p.75-90.

14. Gagliardino JJ. An overview of Argentine contributions to diabetes research in the decade of the 1990s. Diabetes Metab Res Rev 2000;16:43-60.

15. Picena JC, Daniele SM, Arriaga S, Martínez SM, Tarrés MC, Montenegro $\mathbf{S}$ et al. Analogías entre las ratas espontáneamente diabéticas eSS y pacientes diabéticos, con nefropatía y sin retinopatía. Medicina (Buenos Aires) 2002;62:508.

16. Tarrés MC, Montenegro SM, Martínez SM, Picena JC, Toniolo F, Figueroa NS et al. The eSMT rat: a murine model of type 2 human diabetes. Revista de Ciència 1999;23-24:40.

17. Diabetes and Nutrition Study Group of the European Association for the Study of Diabetes. Recommendations for the nutritional management of patients with diabetes mellitus. Diab Nutr Metab 1995; 8:186-9.

18. Ikeda H, Shino A, Matsuo T, Iwatsuka H, Suzuoki Z. A new genetically obese-hyperglycemic rat (Wistar fatty). Diabetes 1981;30:1045-50.

19. Tarrés MC, Martínez SM, Montenegro SM, Picena JC, Naves A, Figueroa $\mathbf{N}$ et al. Estudio a largo plazo de la relación entre dieta, biomasa y expresión de la diabetes en la rata eSS. Medicina (Buenos Aires) 1990;50:235-43.

20. Tarrés MC, Martínez SM, Liborio MM, Picena JC, Rabasa SL. Efecto del ambiente nutricional sobre la expresión del síndrome diabético de las ratas eSS. Medicina (Buenos Aires) 1986;46:429-34.

21. Martínez SM, Tarrés MC, Montenegro SM, Revelant G, Figueroa N, Alonso $\mathrm{D}$ et al. Intermittent dietary restriction in eSS diabetic rats. Effects on metabolic control and skin morphology. Acta Diabetol Lat 1990; 27:329-36.

22. Martínez SM, Tarrés MC, Montenegro SM, Varela M, Torrano A, D'Ottavio AE et al. Effects of sucrose option on the diabetic syndrome of the eSS rat. Isr $\mathrm{J}$ Med Sci 1994;30:761-6.

23. Morton B, Abbot D, Barclay R, Close BS, Ewbank $\mathbf{R}$, Heath $\mathbf{M}$, et al. Removal of blood from laboratory mammals and birds. First report of the BVA/FRAME/ RSPCA/UFAW Joint Working Group on Refinement. Lab Anim 1993;27:1-22.

24. Cheverud JM, Pletscher LS, Vaughn, Marshall B. Differential response to dietary fat in large $(\mathrm{LG} / \mathrm{J})$ and small $(\mathrm{SM} / \mathrm{J})$ inbred mouse strains. Physiol Genomics 1999;1:33-9.
25. Fabens AJ. Properties and fitting of the von Bertalanffy growth curve. Growth 1965;29:265-89.

26. Berkey CS, Laird NM. Non-linear growth curve analysis: estimating the population parameters. Ann Hum Biol 1986;13:111-28.

27. van Dam RM, Rimm EB, Willet WC, Stampfer MJ, Hu FB. Dietary patterns and risk for type 2 diabetes mellitus in US men. Ann Intern Med 2002;136:201-9.

28. Neel JV. The thrifty genotype revisited. In: Kobberling J, Tattersall $R$ (editors). The genetics of diabetes mellitus. London-New York: Academic Press; 1982. p.283-93.

29. Vuguin P, Ma X, Yang X, Surana M, Liu B, Barzilai N. Food deprivation limits insulin secretory capacity in postpubertal rats. Pediatr Res 2001;49:468-73.

30. Shafrir E. Development and consequences of insulin resistance: lessons from animals with hyperinsulinaemia. Diabetes Metab 1996;22:122-31.

31. Surwit RS, Kuhn C. Diet-induced type 2 diabetes and obesity in the C57BL/6J mouse: a stress-related model of human disease En: Shafrir E, editor. Lessons from animal diabetes IV. London: Smith-Gordon and Company Limited Editors; 1993. p.219-28

32. Lebovitz HE, White S, Mikat E, Hackel DB. Control of insulin secretion in the Egyptian Sand Rat (Psammonmys obesus). Diabetologia 1974;10: 679-84.

33. Gutzeit A, Rabinovitch A, Studer P, Trueheart PA, Cerasi E, Renold AE. Decreased intravenous glucose tolerance and low plasma insulin response in spiny mice (Acomys cahirinus). Diabetologia 1974;10:667-70.

34. Shafrir E, Ziv E. Cellular mechanism of nutritionally induced insulin resistance: the desert rodent Psammomys obesus and other animals in which insulin resistance leads to detrimental outcome. J Basic Clin Physiol Pharmacol 1998;9:347-85.

35. Okachi N, Mizuno A, Yoshimoto S, Zhu M, Sano T, Shima K. Is caloric restriction effective in preventing diabetes mellitus in the Otsuka Long Evans Tokushima fatty rat, a model of spontaneous non- insulin- dependent diabetes mellitus. Diabetes Res Clin Pract 1995;27:97106.

36. Ohneda M, Inman LR, Unger RH. Caloric restriction in obese pre-diabetic rats prevents beta cell depletion, loss of beta-cell Glut 2 and glucose incompetence. Diabetologia 1995;38:173-9.

37. Alvarez C, Bailbe D, Picarel-Blanchot F, Bertin E, Pascual-Leone AM, Portha B. Effect of early dietary restriction on insulin action and secretion in the GK rat, a spontaneous model of NIDDM. Am J Physiol Endocrinol Metab 2000;278:1097-103.

38. Ozanne SE, Jensen CB, Tingey KJ, Storgaard H, Madsbad S, Vaag AA. Low birthweight is associated with specific changes in muscle insulin-signalling protein expression. Diabetologia 2005;48:547-52. 
39. Markovic TP, Jenkins AB, Campbell LV, Furler SM, Kraegen EW, Chisholm DJ. The determinants of glycemic responses to diet restriction and weight loss in obesity and NIDDM. Diabetes Care 1998;21:68794.

40. UK Prospective Diabetes Study Group. Response of fasting plasma glucose to diet therapy in newly presenting type II diabetic patients, UKPDS 7 Group. Metabolism 1990;39:905-12.
41. Williams RR. The role of genetics analysis in characterizing obesity. Int J Obesity 1984;8:551-9.

42. Scheen AJ, Paquot N, Jandrain B. How I explore the risk of a patient developing type 2 diabetes. Rev Med Liege 2002;57:113-5.

43. Conlisk AJ, Stein AD, Schroeder DG, Torun B, Grajeda R, Martorell R. Determinants of fasting glucose in young Guatemalan adults. J Am Diet Assoc 2002; 102:109-18. 